\title{
Cost effective localization in distributed sensory networks
}

\author{
Anil Coskun, Hakki Erhan Sevil*, Serhan Ozdemir \\ Artificial Iytelligence and Design Laboratory, Mechanical Engineering Department, Izmir Institute of Technology, 35430 Izmir, Turkey
}

\section{A R T I C L E I N F O}

\section{Article history:}

Received 27 November 2007

Received in revised form

18 May 2010

Accepted 10 October 2010

Available online 9 November 2010

Keywords:

Localization

Distributed sensory networks

Lateral inhibition

Artificial intelligence

\begin{abstract}
A B S T R A C T
The most important mechanism to occur in biological distributed sensory networks (DSNs) is called lateral inhibition, (LI). LI relies on one simple principle. Each sensor strives to suppress its neighbors in proportion to its own excitation. In this study, LI mechanism is exploited to localize the unknown position of a light source that illuminated the photosensitive sensory network containing high and low quality sensors. Each photosensitive sensor was then calibrated to accurately read the distance to the light source. A series of experiments were conducted employing both quality sensors. Low quality array was allowed to take advantage of LI, whereas the high quality one was not. Results showed that the lateral inhibition mechanism increased the sensitivity of inferior quality sensors, giving the ability to make the localization as sensitive as high quality sensors do. This suggests that the networks with multitude of sensors could be made cost-effective, were these sensory networks equipped with $\mathrm{LI}$.
\end{abstract}

(c) 2010 Elsevier Ltd. All rights reserved.

\section{Introduction}

A set of geographically scattered sensors designed to collect information about the environment in which they are deployed is described as a distributed sensory network (DSN). Today many advanced systems employ distributed sensory systems which consist of a large number of sensors in practical applications ranging from aerospace, defense, robotics, automation systems, to monitoring and control of plants. The development and implementation of DSN systems bring about a combination of many different problems in sensor deployment such as network communication, data association, fusion and processing. Although long processing time and the energy needed for processing of information are common problems for DSNs, the primary reason of all these problems that described above and the most important challenge to overcome for DSNs is redundancy. Depending on the increasing number of sensors used in DSN, the input fields of the individual sensors may overlap, leading to redundancy. Although there are many techniques in order to cope with the redundancy problem, lateral inhibition (LI) is one way that stands out for its ubiquity in nature and simplicity. LI is a fundamental peripheral processing in biological systems such as visual, auditory and somatosensory systems. It is commonplace in biological distributed sensory networks. In lateral inhibition mechanism, each individual receptor drives down each of its neighbors in proportion to its own excitation.

\footnotetext{
* Corresponding author. Tel.: +90 232 7506754; fax: +90 2327506701 .

E-mail addresses: erhansevil@iyte.edu.tr (H.E. Sevil), serhanozdemir@iyte.edu.tr (S. Ozdemir).
}

While the first attempts to describe lateral inhibition were made by Ernst Mach on the basis of his perceptual experiments demonstrating Mach bands, Hartline et al. constituted the first statement based on known interactions of biological sensors. The concept of lateral inhibition arose in the extensive experimental research of Hartline and his colleagues on the facetted compound eye of Horseshoe crab (Limulus) (Hartline et al., 1956). The research by Hartline, and later by Barlow (1969), occupied a period of over 50 years and is an outstanding example of bringing quantitative mathematical methods of signal transmission out of complex biological systems. Brooks discussed the features of biological sensors and possible ramifications that might occur, should there be a parameter change.

The most conspicuous feature of LI and localization using sensor networks is the shortage of research over how much advantage LI provides to an existing network. A concrete link between the inclusion of LI and an increase in localization performance is the missing link. One may find numerous examples of elementary study regarding LI both in engineering and biological networks. However one may find almost none of the answers to a set of fundamental questions such as the amount of percent improvement over pinpointing an object in space. This study attempts to answer some. In the light of this shortage, only a few relatively pertinent works are to be presented.

Xie et al. (2002) showed that there is a competition between neurons due to lateral inhibition. This is popularly known as winner-takes-all competition. They set a formula to show the inhibitory connectivity of overlapping groups. Their study stated that it was possible to organize lateral inhibition to mediate the winner-takes-all competition between potentially overlapping groups of neurons. 
Since Hartline and Barlow, there have been plethora publications on LI over neuronal studies about the role of LI within neuron interactions. Exploiting the contrast enhancement aspect of $\mathrm{LI}$ in a general signal processing perspective has rather been very limited. Contrast enhancement is an important area especially in image recognition and related fields. Traditionally, contrast enhancement has been realized by a proper selection of wavelet filters, enhancement functions (Fan and Laine, 1996), multimodal histograms (Grundland and Dodgson, 2006), entropy operators (Khellaf et al., 1991), to name a few.

Two specific examples stand out with the use of LI. One is concerned with an application in image recognition. Edge detection is the focus of this paper which is realized through a neural network having a lateral inhibition ability (Kristensen and Patel, 2002). The second paper is close in content to the current study, in that LI is applied to a two-dimensional photoreceptor array (Wolpert and Micheli-Tzanakou, 1989). The motivation was to analyze the equilibrium of the LI systems. It is claimed that the homeostasis of the system will be inherently unstable. LI is applied by two different configurations. One method is a dynamic algorithm that is applied to a 25-cell rectangular array. A second static method is used on a 19-cell hexagonal array, where both circuits were implemented in VLSI. A hexagonal array is formed in which each node of the source image is directly inhibited by each of its six nearest neighbors. It is stated that hexagonal arrays are not only spatially efficient than their rectangular counterparts but also carry closer resemblance to biological sensory arrays.

The last and the most relevant study is by Cunningham (2007). Researcher tackles the localization problem on a regular and a random $12 \times 12$ array. Localization is realized after a calibration run on statistical inference. Edge and corner detection forms the basis of his study. Unfortunately, it is not clear how the statistical inference is gained and under what assumptions. Even though it is again not quite clear, it is believed that calibration runs are used to pinpoint optimal values of LI parameters. Two interesting real-life examples, however, put an emphasis on the commercial and military potential of LI.

The aim of this study is to show the efficiency of the lateral inhibition mechanism on contrast enhancement, which is applied to target localization. For this purpose, several sensory arrays which contain light detectors with different configurations had been constructed and the unknown position of a light source (target) was estimated by measuring the outputs of these sensory arrays.

To calibrate the system for establishing the link between the sensory output and the distance of the source, the single member of each sensor group (LDRs and photodiodes) was illuminated by adjusting the light source to different positions whose coordinates were known and the response of the sensors were recorded. The next process was to create characteristic curves through regression so that sensor output in volts and the source distance are mapped.
The light source was placed on to a series of unknown locations over the sensory array and the positions of the light source were estimated. Then the lateral inhibition mechanism was applied to the sensor outputs and this process enhanced the response of the sensors. These estimated and modified values were used to find the position of the light source by two computational methods. The first is known as trilateration and the second as $\mathrm{TbHP+}$. Trait-based heterogeneous population plus ( $\mathrm{TbHP}+$ ) is an original algorithm in the training arena, where it uses the general philosophy of combining genetic algorithms (GAs) with simulated annealing (SA) in the very basic principles. More could be found in (Tayfur et al., 2009) on TbHP+.

\section{Lateral inhibition}

Lateral inhibition (LI) is the dominant feature of biological distributed sensory networks where each individual receptor drives down each of its neighbors in proportion to its own excitation. The strengths of these connections are fixed rather than modifiable and are generally arranged as excitatory among nearby receptors and inhibitory among farther receptors. In other words, when any given receptor responds, the excitatory connections tend to increase its response while inhibitory connections try to decrease it. All receptors in the network receive a mixture of excitatory and inhibitory signals from other competitive receptors. As a result of the competitive network structure a distinction between the receptor or a group of receptors which have the strongest output and the receptors with weaker output become larger. Weaker receptors might be suppressed. According to the value of excitatory and inhibitory coefficients and number of interconnected receptors, the response of the whole network can vary. In the case of choosing optimum coefficients, the receptor which has the strongest output suppresses all the other receptor outputs and this kind of a network is called "winner takes all" type.

Fig. 1 defines a set of sensors with lateral inhibition mechanism. The arcs ending in black arrows indicate inhibitory effect of each sensor on the neighboring sensors which are represented by "I". Each sensor has individual input that is designated by " $x_{n}$ " and each sensor drives down the outputs " $y_{n}$ " of its neighbors which are connected to it at black arrow end. The ratio between the amount of sensory output which is driven down by neighbors and the amount of output is called the degree of lateral inhibition (Brooks, 1988).

Effects of lateral inhibition were first recognized in 1886 by the Austrian physicist Ernst Mach who ascertained that all knowledge was based on sensation, and that all scientific observations or measurements were dependent upon the observer's perception. Although the physiological phenomenon discovered by him was not named lateral inhibition, it was the first step for future studies. Mach's study concluded that the brighter and darker contours are

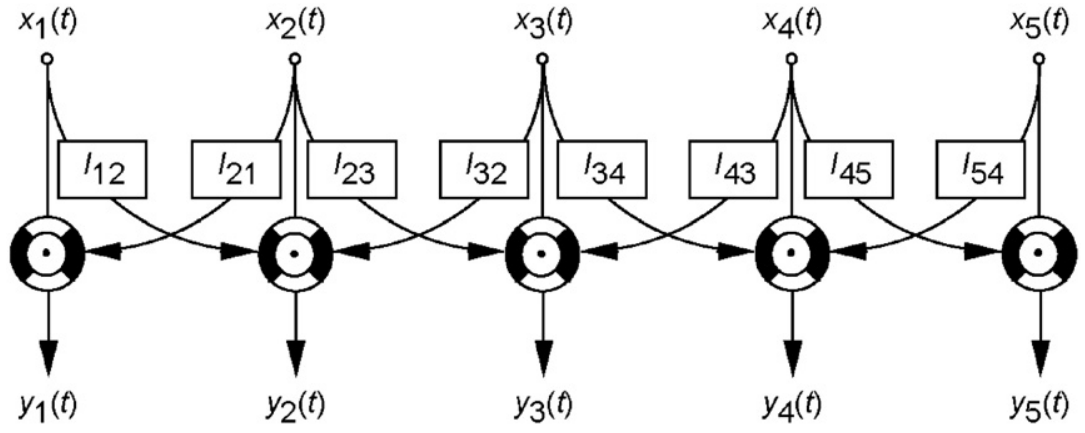

Fig. 1. Schematic illustration of lateral inhibition (Source: www.medical-cybernetics.de). 


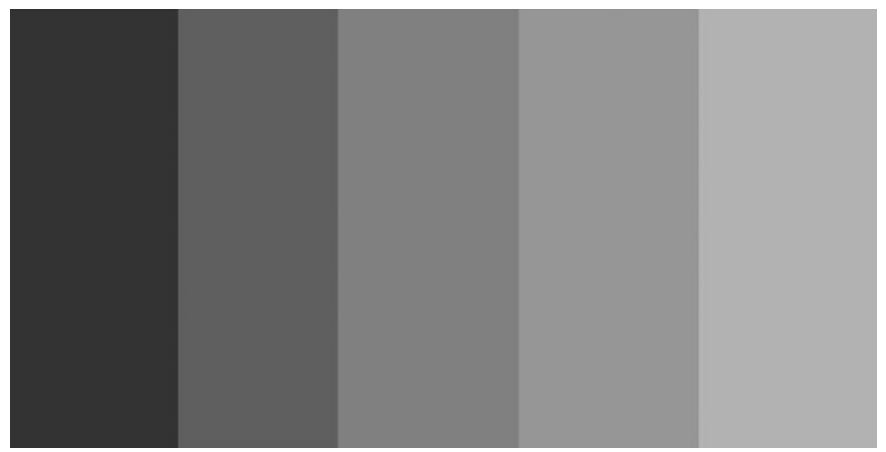

Fig. 2. Mach bands.

physiologically provoked. There is a brightness enhancement at the region where the bright area becomes darker and there is a darker band where the dark area becomes brighter. This effect can be seen in Fig. 2. Although there are two columns which have different but constant physical brightness values near the border line of the two columns, a dissimilarity is perceived by the observer. It is seen that the dark column becomes brighter and bright column becomes darker near the border line.

The most important studies that explained the lateral inhibition mechanism were made by H.K. Hartline in 1934, who received Nobel Prize for this work in 1968. His works were about visual system of Horseshoe crab (Limulus polyphemus) which is a kind of arthropod from North America. The concept of lateral inhibition arose in the comprehensive experimental research of H.K. Hartline and colleagues on the facetted compound eye of Horseshoe crab.

\section{Mathematical model of LI}

Since Hartline, various forms and equations have appeared. Some of the proposed models have accounted for neuronal activities in terms of electrical charges, and down to ion transfers. Some preferred simplified models, representing all main elements of LI. The model below is given in detail in Coskun (2006):

$\frac{d x_{i}}{d t}+x_{i}=\left[b_{i}+\alpha x_{i}^{-}-\beta \sum J_{i j} x_{j}^{-}\right]^{+}$

Eq. (1) gives the dynamics of the network. This equation defines the effect of lateral inhibition where; $J_{i j}$ is the lateral inhibition between the neurons $i$ and $j, \alpha$ the strength of self-excitation $(\alpha>0), \beta$ the strength of lateral inhibition $(\beta>0), b$ the external input, $x_{i}$ the activity of neuron, $x_{i}^{-}$the raw activity of neuron and $d x_{i} / d t$ the change in the activity of $i$ th neuron (sensor).

Furthermore, the value $J$ is calculated as follows:

$J_{i j}=\prod_{a=1}^{m}\left(1-\xi_{i}^{a} \xi_{j}^{a}\right)= \begin{cases}0 & f i \text { and } j \text { both belong to a group } \\ 1 & \text { otherwise }\end{cases}$

where

$\xi_{i}^{a}= \begin{cases}1 & f i \text { th neuron is in ath group } \\ 0 & \text { otherwise }\end{cases}$

The steady state of Eq. (1) is Eq. (3), where for a given set of parameters, and a constant input, output of the sensor is timeinvariant as below:

$x_{i}=x_{i}^{-}-\beta_{1} \sum x_{i j}+\alpha x_{i}^{-}$

$\alpha$ is the strength of self-excitation ( $\alpha>0), \beta$ the strength of lateral inhibition $(\beta>0), x_{i}$ the final activity of sensor, $x_{i j}$ the activity of sensors which are in the neighborhood of $x_{i}$.

\section{Experimental setup}

To observe the effects of lateral inhibition mechanism, two experimental setups were built. Each experimental setup consists of a light source and a sensory array with several photosensitive sensors such as photodiodes because of their low cost. One problem encountered early in the experiments was the memory effect with the LDRs. LDRs did "remember" the recent trials, giving inaccurate results. This is bypassed with the use of photodiodes throughout the trials.

In the first setup (Fig. 3), there is a board containing several photosensitive sensors. These sensors were stimulated by a pair of point light sources whose position in space could be adjusted as seen on the left-hand side of Fig. 3. The second experimental setup consists of a light source; $12 \mathrm{~V} / 20 \mathrm{~W}$ halogen lamp and a sensory array of several photosensitive sensors. The light source and photosensitive array were placed within an aluminum frame. This frame stabilized the sensory array and gave the light source an ability to move in three dimensions, and also the whole setup could be covered to allow a dark chamber to minimize the lightinduced noise (Fig. 4(a, b)). To control the experiment, the same sensor is used for both high and low quality sensors. What made the same sensor a "low" quality one is a pane of glass with soot smeared all over the surface kept above the sensors. The amount of soot was determined by trial and error so as to degrade sensor performance but not to block the light completely.

These sensors were connected to a Pentium 4 PC over a multiplexer which was then interfaced with a Keithley 2750 datalogger instrument and this instrument was controlled via ExceLink software (Fig. 5). Each sensor had an address and successive readings were made in turn, over a specific setup.

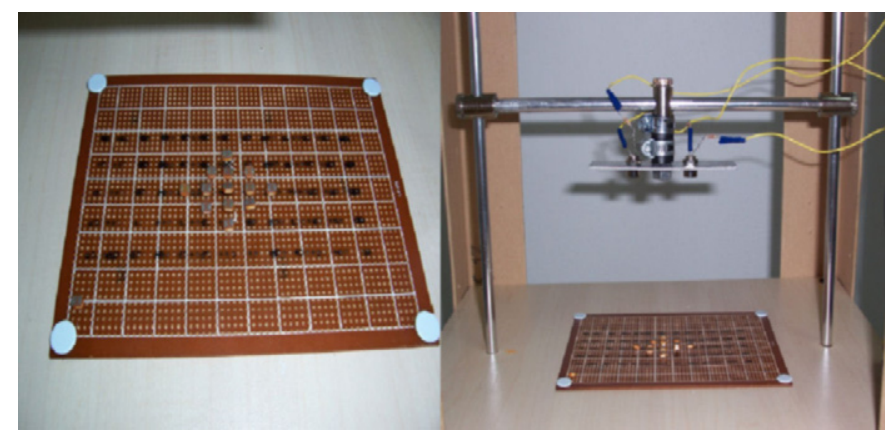

Fig. 3. The first experiment setup. a

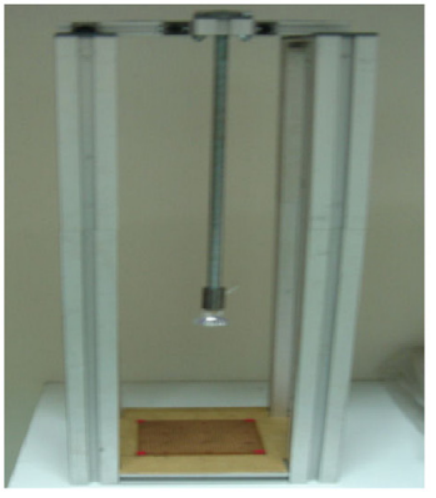

b

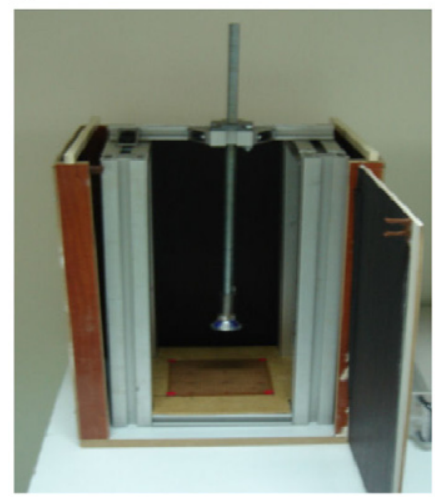

Fig. 4. (a, b) The second experiment setup. 


\section{Lateral inhibition on localization}

In the experimental process, the voltage values from photodiodes were processed and transferred into a distance reading. These distances were then used to localize the light source. For this localization process the trilateration method and a GA algorithm (TbHP+) were used. The same process was conducted for each photodiode array by applying lateral inhibition mechanism.

Trilateration is a basic geometric principle that helps find a location using relative positions of three or more known locations a

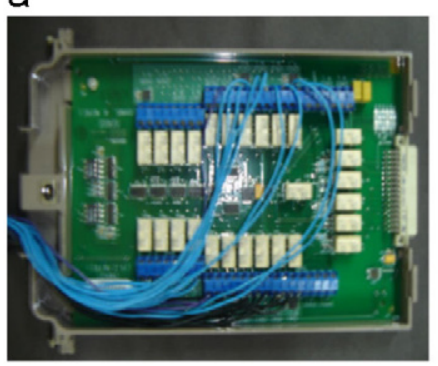

b

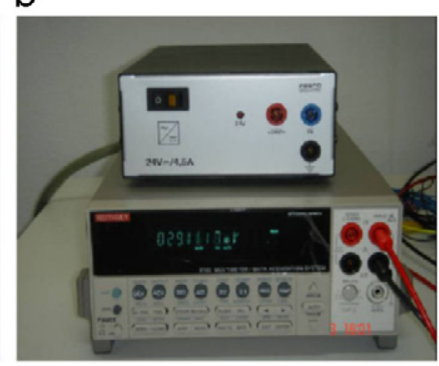

Fig. 5. (a) Connections of multiplexer and (b) Keithley datalogger. similar to triangulation. Unlike triangulation, which uses angle measurements (together with at least one known distance) to calculate the subject's location, trilateration uses the known locations of two or more reference points, and the measured distance between the subject and each reference point. To accurately and uniquely determine the relative location of a point in two-dimensional space, at least three circles are needed to pinpoint the location. In three-dimensional space, at least four spheres are needed. Trilateration can be applied to many different areas, such as geographic mapping, navigation (e.g. GPS systems), and for the progress in electronic distance-measuring it is preferred to triangulation.

In lateral inhibition, it is assumed that each photodiode has an interconnection with the three closest neighbor photodiodes. Inhibition coefficient $(\beta)$ was chosen 0.05 and the excitation coefficient $(\alpha)$ was chosen 0.145 . As a first step an array which contains $9(3 \times 3)$ high quality photodiodes was constructed. The $X$, $Y$ and $Z$ coordinates of the light source were adjusted to 41,45 and $200 \mathrm{~mm}$, respectively. The coordinates of photodiodes and the measurement results are tabulated in Table 1 . The result of localization process with high quality photodiode array can be seen in Table 2. After the localization, the estimated and the real coordinates of the light source were compared. The result of this comparison is given in Table 4 . The results of applying lateral

Table 1

Results of measurement with 9 high quality photodiodes.

\begin{tabular}{|c|c|c|c|c|c|c|c|}
\hline \multirow[t]{2}{*}{ Photodiode number } & \multicolumn{3}{|c|}{ Coordinates } & \multirow{2}{*}{$\begin{array}{l}\text { Measured } \\
\text { voltage }(\mathbf{V})\end{array}$} & \multirow{2}{*}{$\begin{array}{l}\text { Estimated total } \\
\text { distance }(\mathrm{mm})\end{array}$} & \multirow[t]{2}{*}{ Voltage after LI (V) } & \multirow{2}{*}{$\begin{array}{l}\text { Estimated total } \\
\text { distance after } \mathrm{LI}(\mathrm{mm})\end{array}$} \\
\hline & $X$ & $\boldsymbol{Y}$ & $Z$ & & & & \\
\hline 1 & 0 & 45 & 0 & 0.5903 & 194.46 & 0.5874 & 203.31 \\
\hline 2 & 41 & 45 & 0 & 0.5915 & 191.18 & 0.5887 & 199.35 \\
\hline 3 & 82 & 45 & 0 & 0.5901 & 195.13 & 0.5871 & 204.14 \\
\hline 4 & 0 & 80 & 0 & 0.5892 & 197.96 & 0.5862 & 206.91 \\
\hline 5 & 41 & 80 & 0 & 0.5905 & 194.07 & 0.5877 & 202.48 \\
\hline 6 & 82 & 80 & 0 & 0.5893 & 197.61 & 0.5864 & 206.5 \\
\hline 7 & 0 & 115 & 0 & 0.5863 & 206.85 & 0.5829 & 217.72 \\
\hline 8 & 41 & 115 & 0 & 0.588 & 201.57 & 0.585 & 210.66 \\
\hline 9 & 82 & 115 & 0 & 0.5867 & 205.6 & 0.5833 & 216.23 \\
\hline
\end{tabular}

Table 2

Results of comparison of high quality array and low quality array.

\begin{tabular}{|c|c|c|c|c|c|c|c|c|c|}
\hline \multirow[t]{2}{*}{ Sensor type } & \multicolumn{3}{|c|}{ Real coordinates } & \multicolumn{3}{|c|}{ TbHP+ } & \multicolumn{3}{|c|}{ Trilateration } \\
\hline & $\boldsymbol{X}$ & $\boldsymbol{Y}$ & $Z$ & $\boldsymbol{X}$ & $\boldsymbol{Y}$ & $Z$ & $\boldsymbol{X}$ & $\boldsymbol{Y}$ & $Z$ \\
\hline High quality sensors without LI & 41 & 45 & 200 & 37.79 & 39.21 & 189.01 & 35.93 & 56.74 & 195.19 \\
\hline High quality sensors with LI & 41 & 45 & 200 & 42.06 & 43.75 & 199.57 & 39.99 & 46.19 & 202.65 \\
\hline Low quality sensors without LI & 41 & 45 & 200 & 34.04 & 33.62 & 178.1 & 28.14 & 63.51 & 194.09 \\
\hline Low quality sensors with LI & 41 & 45 & 200 & 43.18 & 42.43 & 188.65 & 29.03 & 58.96 & 198.92 \\
\hline
\end{tabular}

Table 3

Results of measurement with 9 low quality photodiodes.

\begin{tabular}{|c|c|c|c|c|c|c|c|}
\hline \multirow{2}{*}{$\begin{array}{l}\text { Photodiode } \\
\text { Number }\end{array}$} & \multicolumn{3}{|c|}{ Coordinates } & \multirow{2}{*}{$\begin{array}{l}\text { Measured } \\
\text { voltage }(V)\end{array}$} & \multirow{2}{*}{$\begin{array}{l}\text { Estimated total } \\
\text { distance }(\mathrm{mm})\end{array}$} & \multirow[t]{2}{*}{ Voltage after LI (V) } & \multirow{2}{*}{$\begin{array}{l}\text { Estimated total } \\
\text { distance after } \mathrm{LI}(\mathrm{mm})\end{array}$} \\
\hline & $\boldsymbol{X}$ & $Y$ & $Z$ & & & & \\
\hline 1 & 0 & 45 & 0 & 0.29218 & 187.16 & 0.29081 & 190.9 \\
\hline 2 & 41 & 45 & 0 & 0.29281 & 185.48 & 0.29148 & 189.06 \\
\hline 3 & 82 & 45 & 0 & 0.29177 & 188.27 & 0.29034 & 192.21 \\
\hline 4 & 0 & 80 & 0 & 0.29018 & 192.64 & 0.28872 & 196.75 \\
\hline 5 & 41 & 80 & 0 & 0.29178 & 188.24 & 0.29051 & 191.71 \\
\hline 6 & 82 & 80 & 0 & 0.29022 & 192.53 & 0.28878 & 196.6 \\
\hline 7 & 0 & 115 & 0 & 0.28666 & 202.77 & 0.2847 & 208.69 \\
\hline 8 & 41 & 115 & 0 & 0.28852 & 197.34 & 0.28708 & 201.51 \\
\hline 9 & 82 & 115 & 0 & 0.28696 & 201.88 & 0.28504 & 207.65 \\
\hline
\end{tabular}


inhibition to localization process for the same array are also given in Table 2. Comparison of the real coordinates and the estimated coordinates of the light source after applying lateral inhibition mechanism are seen in Table 4.

The localization was repeated with an array containing low quality photodiodes. The number of photodiodes was kept the same as before. The localization with the low quality units was done with and without lateral inhibition mechanism. The coordinates of photodiodes and the measurement results are shown in Tables 1-3.

The effects of the sensor number on the localization ability might be better realized with an array which contained $16(4 \times 4)$ low quality photodiodes. At first, the coordination of the light source was computed without applying LI. Then the same process was repeated with LI. The coordinates of photodiodes and the measurement results are shown in Table 5. The results of the localization processes are shown in Tables 6 and 7 .

It is clearly seen in Tables 3 and 6 that by LI, estimates converge to the real coordinates. Although the same number and the same quality photodiodes were used for both measurements, the

Table 4

Error comparison of high quality array and low quality array.

\begin{tabular}{|c|c|c|c|c|c|c|}
\hline \multirow[t]{3}{*}{ Sensor type } & \multicolumn{6}{|c|}{ Error (\%) } \\
\hline & \multicolumn{3}{|c|}{ TbhP+ } & \multicolumn{3}{|c|}{ Trilateration } \\
\hline & $\boldsymbol{X}$ & $\boldsymbol{Y}$ & $Z$ & $\boldsymbol{X}$ & $\boldsymbol{Y}$ & $Z$ \\
\hline High quality sensors without LI & 7.83 & 12.85 & 5.49 & 12.37 & 26.08 & 2.40 \\
\hline High quality sensors with LI & 2.58 & 2.79 & 0.21 & 2.46 & 2.64 & 1.33 \\
\hline Low quality sensors without LI & 16.98 & 25.29 & 10.95 & 31.37 & 41.13 & 2.96 \\
\hline Low quality sensors with LI & 5.32 & 5.71 & 5.68 & 29.20 & 31.02 & 0.54 \\
\hline
\end{tabular}

deviation in the localization of the position of the light source decreased. It proves that lateral inhibition mechanism enhances localization. In addition, applying LI to sensory arrays with high in number and low in quality photodiodes provides localization as sensitive as the array which contains less in number but high in quality photodiodes. It is clearly seen in Table 4 that in the case of using low quality array, the error values for the $X$-axis $11.77 \%$ and the $Z$-axis $6.62 \%$ are kept close to the error value $7.84 \%$ for the $X$-axis and $5.49 \%$ for the $Z$-axis which was obtained by using high quality sensory array without lateral inhibition mechanism. Similarly for the $Y$-axis, low quality sensory array proved better (1.53\%) than high quality sensory array as a result of lateral inhibition mechanism.

\section{Conclusions}

In this study, the potential help in sensing by LI which is also known as the contrast enhancement mechanism by biologists was examined. Lateral inhibition is a basic data processing principle for biological systems and it is commonplace for biological DSNs including human sensory and nervous systems. The interactions

Table 7

. Error comparison of 9 high quality photodiodes and 16 low quality photodiodes.

\begin{tabular}{|c|c|c|c|c|c|c|}
\hline \multirow[t]{3}{*}{ Sensor type } & \multicolumn{6}{|c|}{ Error (\%) } \\
\hline & \multicolumn{3}{|c|}{ TbhP+ } & \multicolumn{3}{|c|}{ Trilateration } \\
\hline & $\boldsymbol{X}$ & $Y$ & $Z$ & $\boldsymbol{X}$ & $\boldsymbol{Y}$ & $Z$ \\
\hline 9 high quality sensors without $\mathrm{LI}$ & 7.83 & 12.85 & 5.49 & 12.37 & 26.09 & 2.41 \\
\hline 16 low quality sensors without LI & 16.93 & 18.78 & 9.92 & 27.63 & 28.67 & 7.63 \\
\hline 16 low quality sensors with LI & 11.77 & 1.53 & 6.62 & 14.10 & 4.33 & 2.48 \\
\hline
\end{tabular}

Table 5

Results of measurement with 16 low quality photodiodes.

\begin{tabular}{|c|c|c|c|c|c|c|c|}
\hline \multirow{2}{*}{$\begin{array}{l}\text { Photodiode } \\
\text { Number }\end{array}$} & \multicolumn{3}{|c|}{ Coordinates } & \multirow{2}{*}{$\begin{array}{l}\text { Measured } \\
\text { voltage }(\mathbf{V})\end{array}$} & \multirow{2}{*}{$\begin{array}{l}\text { Estimated total } \\
\text { distance }(\mathrm{mm})\end{array}$} & \multirow{2}{*}{$\begin{array}{l}\text { Voltage } \\
\text { after LI (V) }\end{array}$} & \multirow{2}{*}{$\begin{array}{l}\text { Estimated total } \\
\text { distance after } \mathrm{LI}(\mathrm{mm})\end{array}$} \\
\hline & $\boldsymbol{X}$ & $Y$ & $Z$ & & & & \\
\hline 1 & 0 & 45 & 0 & 0.29319 & 184.46 & 0.29164 & 188.62 \\
\hline 2 & 27.3 & 45 & 0 & 0.2948 & 180.28 & 0.29342 & 183.86 \\
\hline 3 & 54.6 & 45 & 0 & 0.29544 & 178.64 & 0.29418 & 181.87 \\
\hline 4 & 82 & 45 & 0 & 0.29319 & 184.46 & 0.2916 & 188.74 \\
\hline 5 & 0 & 68.3 & 0 & 0.29275 & 185.63 & 0.29138 & 189.33 \\
\hline 6 & 27.3 & 68.3 & 0 & 0.29378 & 182.92 & 0.29233 & 186.75 \\
\hline 7 & 54.6 & 68.3 & 0 & 0.29398 & 182.39 & 0.29257 & 186.11 \\
\hline 8 & 82 & 68.3 & 0 & 0.29275 & 185.63 & 0.29137 & 189.36 \\
\hline 9 & 0 & 91.6 & 0 & 0.28947 & 194.64 & 0.28789 & 199.15 \\
\hline 10 & 27.3 & 91.6 & 0 & 0.29214 & 187.26 & 0.2908 & 190.93 \\
\hline 11 & 54.6 & 91.6 & 0 & 0.29254 & 186.19 & 0.29139 & 189.31 \\
\hline 12 & 82 & 91.6 & 0 & 0.28947 & 194.64 & 0.28787 & 199.2 \\
\hline 13 & 0 & 114.9 & 0 & 0.28604 & 204.62 & 0.28404 & 210.73 \\
\hline 14 & 27.3 & 114.9 & 0 & 0.28784 & 199.31 & 0.28626 & 203.95 \\
\hline 15 & 54.6 & 114.9 & 0 & 0.28804 & 198.72 & 0.28648 & 203.29 \\
\hline 16 & 82 & 114.9 & 0 & 0.28604 & 204.62 & 0.28401 & 210.82 \\
\hline
\end{tabular}

Table 6

Result comparison of 9 high quality photodiodes and 16 low quality photodiodes.

\begin{tabular}{|c|c|c|c|c|c|c|c|c|c|}
\hline \multirow[t]{2}{*}{ Sensor type } & \multicolumn{3}{|c|}{ Real Coordinates } & \multicolumn{3}{|c|}{ TbHP+ } & \multicolumn{3}{|c|}{ Trilateration } \\
\hline & $\boldsymbol{X}$ & $\boldsymbol{Y}$ & $Z$ & $\boldsymbol{X}$ & $\boldsymbol{Y}$ & $Z$ & $\boldsymbol{X}$ & $\boldsymbol{Y}$ & $Z$ \\
\hline 9 high quality sensors without LI & 41 & 45 & 200 & 37.79 & 39.21 & 189.01 & 35.93 & 56.74 & 195.19 \\
\hline 16 low quality sensors without LI & 41 & 45 & 200 & 34.06 & 36.55 & 180.15 & 52.33 & 32.10 & 184.75 \\
\hline 16 low quality sensors with LI & 41 & 45 & 200 & 45.83 & 45.69 & 186.76 & 46.78 & 43.05 & 195.04 \\
\hline
\end{tabular}


within LI can be excitatory or inhibitory. As a result of it, each sensor drives down the neighboring sensors in proportion to its own value. It has an important role for human vision, audition and somatic sensation.

An experimental setup for getting the effects of lateral inhibition was built up. It consisted of photodiodes and an adjustable light source. It was observed that, the same effects of lateral inhibition which play important role for biological systems are also at work for the ad hoc setup. Each sensor was calibrated, and the output of each sensor was employed to localize the position of the light source. Localization process was done by trilateration and TbHP+ algorithm.

Results showed that lateral inhibition mechanism increased the sensitivity of the localization. When the estimated coordinates and the real ones of the light source were compared, it was observed that the lateral inhibition for identical sensory network with 9 $(3 \times 3)$ high quality sensors reduced error values from $7.83 \%$, $12.85 \%, 5.49 \%$ to $2.58 \%, 2.79 \%, 0.21 \%$ for the $X, Y$ and $Z$ axes, respectively. This process was repeated with another network that contains $16(4 \times 4)$ low quality and low cost sensors. It was seen that the result of positioning by low quality sensors with LI converged to the result which were taken by using high quality sensory network without lateral inhibition. The error values for low quality sensory array decreased by $16.93 \%, 18.78 \%, 9.92 \%$ to $11.77 \%$, $1.53 \%$ and $6.62 \%$ for the $X, Y$ and $Z$ axes, respectively. For high quality sensory network the error values were calculated as $7.83 \%$, $12.85 \%, 5.49 \%$ for the $X, Y$ and $Z$ axes, respectively.

LI has a great potential for the artificial intelligence related fields and for general signal processing. The competition among sensors has benefits, such as the reduction of redundancy, and contrast enhancement. DSNs must become economically viable since more and more intelligence related applications require an abundance of sensing units. Authors believe that $\mathrm{LI}$ is not standing at its rightful niche. The understanding of this rather less-known algorithm by wider circles could hopefully help process signals faster and more efficiently.

\section{References}

Barlow, R.B., 1969. Inhibitory fields in the limulus lateral eye. Journal of General Physiology 54, 383-397.

Brooks, M., 1988. Highly redundant sensing in robotics-analogies from biology: distributed sensing and learning. In: Proceedings of the NATO Advanced Research Workshop on Highly Redundant Sensing in Robotic Systems, Italy.

Coskun, A., 2006. Contrast enhancement by lateral inhibition in a sensory network M.Sc. Thesis, Izmir Institute of Technology, Mechanical Engineering Department.

Cunningham, H.A., 2007. Sensor localization with lateral inhibition and statistical inference. In: Proceedings of the IEEE SECON, California, USA, 18-21 June 2007, pp. 70-72.

Fan, J., Laine, A., 1996. Contrast enhancement by multiscale and nonlinear operators. In: Aldroubi, A., Unser, M. (Eds.), Wavelets in Medicine and Biology. CRC Press, Florida, USA, pp. 163-189.

Grundland, M., Dodgson, N.A. 2006. Automatic contrast enhancement by histogram warping. In: K. Wojciechowski, B. Smolka, H. Palus, R.S. Kozera, W. Skarbek, L. Noakes (Eds.), Computer Vision and Graphics: International Conference, ICCVG 2004, Warsaw, Poland, September 2004, Proceedings (Computational Imaging and Vision).

Hartline, H.K., Wagner, H.G., Ratliff, F., 1956. Inhibition in the eye of limulus. Journal of General Physiology 39, 651-673.

Khellaf, A., Bbeghdadi, A., Dupoisot, H., 1991. Entropic contrast enhancement. IEEE Transactions on Medical Imaging 10, 589-592.

Kristensen, T., Patel, R., 2002. Edge detection in a lateral inhibition network. In: Proceedings of the 2002 International Joint Conference on Neural Networks, Honolulu, HI, USA, 12-17 May 2002, pp. 1620-1624.

Tayfur, G., Sevil, H.E., Gezgin, E., Ozdemir, S., 2009. Trait-based heterogeneous populations plus (TbHP+) genetic algorithm. Mathematical and Computer Modelling 49 (3-4), 709-720.

Wolpert, S., Micheli-Tzanakou, E., 1989. Experiments with lateral inhibition in twodimensional photoreceptor arrays. In: Proceedings of the 1989 Fifteenth Annual Northeast Bioengineering Conference, Boston, MA, USA, 27-28 March 1989, pp. 181-182.

Xie, X., Hahnloser, H.R.R., Seung, S.H., 2002. Selectively grouping neurons in recurrent networks of lateral inhibition. Neural Computation 14, 2627-2646. 